\title{
Traumatic diaphragmatic injuries: a retrospective review of a 12-year experience at a tertiary trauma centre
}

Beng Leong $\underline{\mathrm{Lim}}^{1}$, MBBS, MRCS, Li Tserng $\underline{T \mathrm{Te}^{2}}$, MBBS, FRCS, Ming Terk $\underline{C h i u^{2}}$, MBBS, FRCS, Marxengel L A sinas-Tan $^{1}$, MD, MPH, Eillyne $\underline{\text { Seow }}^{3}$, MBBS, FRCS

INTRODUCTION Traumatic diaphragmatic injuries (TDIs) are clinically challenging. We aimed to review TDIs treated at a tertiary trauma centre over a 12-year period.

METHODS This was a single-centre retrospective review of adult patients with TDls treated between 1 January 2003 and 31 December 2014. Primary outcomes were mortality rates and Injury Severity Scores (ISS) associated with each TDI subtype. Secondary outcomes included proportions of TDIs diagnosed radiologically, operatively or during autopsy. We compared the TDI subtypes with respect to mechanism of injury, mortality rates and median ISS. Data was analysed using descriptive statistics.

RESULTS Among 46 patients studied, the TDI subtypes noted were acute diaphragmatic herniation $(n=14,30.4 \%)$, tears $(n=22,47.8 \%)$ and contusions $(n=10,21.7 \%)$. Patients with these TDI subtypes had a mortality rate of $35.7 \%-100 \%$, while the ISS ranges for survivors and deaths were 22.0-34.0 (interquartile range [IQR] 6.5-23.0) and 53.5-66.0 (IQR 16.0-28.5), respectively. TDIs were identified via chest radiography $(n=2 / 33,6.1 \%)$ and computed tomography $(n=6 / 13,46.2 \%)$. All survivors $(n=21)$ and deaths $(n=25)$ underwent open surgery or autopsy, respectively, which confirmed TDIs. Blunt traumas and penetrating traumas were more frequently associated with acute herniation/contusions and tears, respectively. There were statistically significant differences among the TDI subtypes in their mechanism of injury, mortality rate and median ISS of survivors.

CONCLUSION TDIs showed varying injury patterns with blunt versus penetrating mechanisms of injury, and were associated with significant mortality rates. Preoperative imaging had limited diagnostic use.

Keywords: mortality, traumatic diaphragmatic injuries

\section{INTRODUCTION}

Traumatic diaphragmatic injuries (TDIs) can result in the disappearance of the thoracoabdominal pressure gradient and allow abdominal viscera to migrate into the thorax. ${ }^{(1)}$ This migration can alter respiratory mechanics, compress the vena cava and reduce venous return to the heart, and result in respiratory failure and circulatory collapse. ${ }^{(2)}$ TDIs can result from blunt or penetrating injuries. Their prevalence varies in the many series reported in the literature, likely because their rates can be under- or over-reported. Patients with TDIs reportedly make up $0.5 \%-1.6 \%$ of patients admitted for blunt trauma. ${ }^{(3)}$ Shah et al, on the other hand, reported that up to $8 \%$ of all patients undergoing a laparotomy or thoracotomy for trauma would have an incidental finding of diaphragmatic injury. ${ }^{(4)}$

The spectrum of TDIs ${ }^{(5-7)}$ ranges from contusions and lacerations to major defects, with abdominal organ herniation. Clinical presentations are widely variable. TDIs from diaphragmatic contusions and lacerations can be asymptomatic. They can also present as a state of haemodynamic instability due to bleeding from other concomitant injuries, or as a delayed manifestation of intestinal obstruction and respiratory involvement due to diaphragmatic hernia, months or even years after the actual trauma. ${ }^{(8,9)}$

TDIs remain a diagnostic and therapeutic challenge for the emergency physician and trauma surgeon. They are often associated with thoracic and abdominal injuries that can be life-threatening and distract the attention of the clinician from the diaphragm. These concomitant serious injuries and the lack of accurate diagnostic imaging studies for the diaphragm ${ }^{(1)}$ make diagnosis and treatment difficult.

The aims of our study were to review the spectrum and outcomes of TDIs in a busy metropolitan and tertiary trauma centre, as well as to emphasise the difficulties faced in managing these injuries.

\section{METHODS}

We conducted a retrospective cohort study of adult patients with TDIs treated at Tan Tock Seng Hospital (TTSH), Singapore, using its trauma data registry. The study was approved by the National Healthcare Group ethics committee. TTSH is a 1,500bed adult tertiary hospital that receives referrals from all regions of Singapore. The trauma service at the hospital maintains a prospective trauma registry that captures all traumatic arrest patients attended to by its emergency department (ED) and nonarrest patients referred to the trauma service by the ED based on an existing activation protocol. These activation criteria are shown in Box 1.

Our study included patients aged $\geq 16$ years and who had evidence of TDIs captured in the registry from 1 January 2003 to

${ }^{1}$ Emergency Department, Ng Teng Fong General Hospital, ${ }^{2}$ Trauma and Acute Care Surgery, Department of General Surgery, ${ }^{3}$ Emergency Department, Tan Tock Seng Hospital, Singapore

Correspondence: Dr Lim Beng Leong, Consultant, Emergency Department, Ng Teng Fong General Hospital, 1 Jurong East Street 21 , Singapore 609606. Beng_Leong_Lim@juronghealth.com.sg 


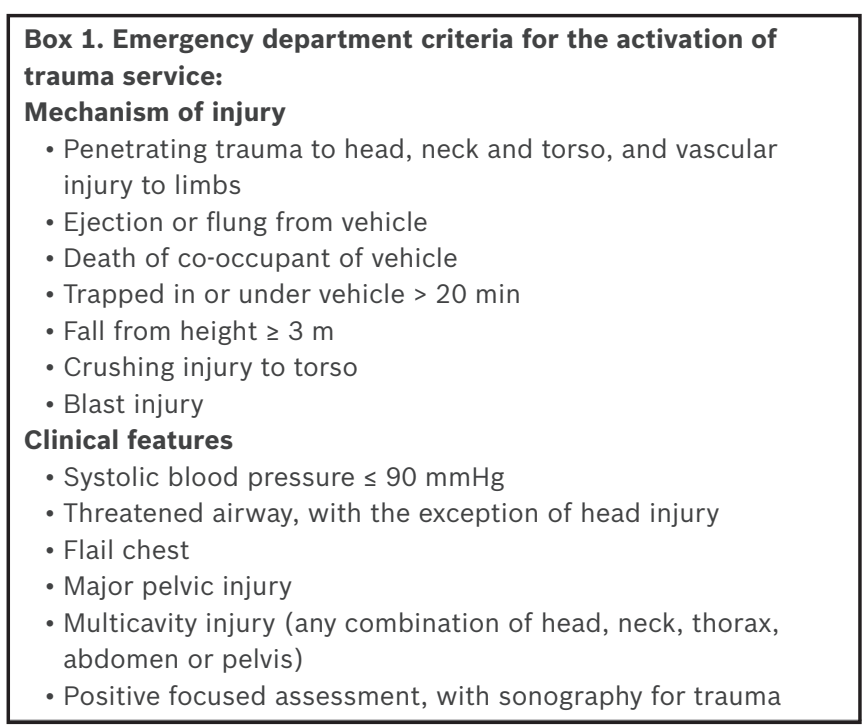

31 December 2014. Evidence of TDIs could be found in the ED and trauma service clinical notes, radiological reports, operative findings and post-mortem records that were captured by the trauma registry. Radiological reports included chest radiography and helical computed tomography (CT) imaging reported by our hospital's radiologists. Operative findings included notes indicating the operative procedures performed and specific intraoperative findings. Post-mortem records were formal autopsy reports by national pathologists to whom patients who died were referred. Two investigators reviewed patients identified by the trauma registry and supplemented data collection using the hospital and national electronic databases. They also searched these databases, for periods ranging from six months to ten years after discharge, for records of any re-attendances at Singapore's public hospitals on account of delayed manifestations of TDIs. Data on demographic, clinical, radiological, operative and postmortem findings was collected.

There are differences between centres in their approach toward managing non-arrest penetrating torso injuries, especially for haemodynamically stable patients. Our trauma service adopts the following principles for managing such patients upon referral from the ED. Indications for immediate open surgery (e.g. thoracotomy and/or laparotomy) include gunshot wounds, haemodynamic instability, continuous bleeding from the chest or abdomen, clinical evidence of peritonitis, evisceration, and radiological findings of free intraperitoneal air and diaphragmatic herniation. For patients with penetrating non-gunshot torso trauma who are haemodynamically stable and have no signs of peritonitis, we adopt an approach of selective conservatism. For these patients with thoracoabdominal penetrating injuries, contrast CT is performed to determine the trajectory, evidence of peritoneal breach and intra-abdominal injuries characterised by free intraperitoneal fluid or air. If there is suspicion or evidence of peritoneal breach or intra-abdominal injuries on contrast CT, laparoscopy is performed. If a diaphragmatic breach is confirmed, it will be repaired either laparoscopically or via laparotomy depending on the individual surgeon's preference and other laparoscopic findings. Stable patients with anterior abdominal wounds undergo surgical exploration to determine any breach in the anterior fascia. An exploration with negative findings is reliable and highly sensitive. ${ }^{(10,11)}$ If the anterior fascia is breached, a diagnostic laparoscopy is performed to determine peritoneal penetration; if peritoneal penetration occurs, an exploratory laparotomy will follow. Stable patients with posterior abdominal or flank wounds undergo contrast $\mathrm{CT}$ of the abdomen to determine the trajectory, peritoneal breach or intra-abdominal injuries. Further management (i.e. laparoscopy vs. laparotomy) of patients is dictated by CT findings. Stable patients with thoracic wounds are observed following chest radiography, with further imaging being decided upon based on the individual clinician's judgment.

The primary outcomes of this study were mortality rates associated with each subtype of TDI together with their respective Injury Severity Scores (ISS) among survivors and deaths. Secondary outcomes included the proportions of patients with the different subtypes of TDIs diagnosed radiologically (via chest radiography or CT), operatively (on open or laparoscopic surgery) or postmortem, and the distribution of the spectrum of TDIs based on the mechanism of injury (i.e. blunt vs. penetrating). In addition, we also compared the different subtypes of TDIs with respect to their mechanism of injury (blunt vs. penetrating), mortality rates, and median ISS of survivors and deaths.

Data was analysed using descriptive statistics with IBM SPSS Statistics version 21.0 (IBM Corp, Armonk, NY, USA). Findings were reported as median and interquartile range (IQR). Fisher's exact test was used to compare categorical variables, and MannWhitney $U$ test and Kruskal-Wallis test were utilised to compare continuous data. The $p$-values were two-tailed and $p<0.05$ was considered to be statistically significant.

\section{RESULTS}

Among 13,130 records of patients captured by our trauma registry, $46(0.4 \%)$ patients were treated for TDIs during the study period. There was no missing data. Table I shows the baseline characteristics of the study population.

TDIs were on the left side for $37(80.4 \%)$ patients and right side for $9(19.6 \%)$ patients. Patients were found to have three broad subtypes of TDIs: acute diaphragmatic herniation $(\mathrm{n}=14$, $30.4 \%$ ); tears ( $n=22,47.8 \%)$; and contusions ( $n=10,21.7 \%$ ). No patients had chronic diaphragmatic herniation. Table II shows the distribution of the TDI subtypes according to the mechanism of injury.

Among patients with acute diaphragmatic herniation $(n=14)$, there were $9(64.3 \%)$ survivors and $5(35.7 \%)$ deaths. The median age was 35.0 (IQR 21.0) years. Among the survivors $(n=9)$, the median ISS was 34.0 (IQR 23.0). All of the nine survivors received chest radiography. 2 (22.2\%) chest radiographs revealed stomach and bowel herniation, both of which were left sided. CT of the thorax and CT of the abdomen/pelvis (CTAP) was performed for the remaining $7(77.8 \%)$ survivors, and $1(11.1 \%)$ patient had repeat CTAP. CT was not performed for the two survivors for whom chest radiography had already revealed diaphragmatic herniation. Among the seven patients for whom CT was performed, 4 (57.1\%) had evidence of diaphragmatic tear 
and herniation, all of which were on the left. One of these four patients had normal initial CT thorax and CTAP, and only showed evidence of diaphragmatic tear and herniation on repeat CTAP.

All nine survivors underwent laparotomy, and 1 (11.1\%) underwent both laparotomy and thoracotomy. All except one patient had surgery within four hours of ED consultation. The remaining patient, who had a time lapse of eight days between

Table I. Baseline characteristics of patients with traumatic diaphragmatic injuries $(n=46)$.

\begin{tabular}{|c|c|}
\hline Characteristic & No. (\%) \\
\hline Age $(y r)^{*}$ & $36.5(30.5)$ \\
\hline \multicolumn{2}{|l|}{ Gender } \\
\hline Men & $37(80.4)$ \\
\hline Women & $9(19.6)$ \\
\hline \multicolumn{2}{|l|}{ Ethnicity } \\
\hline Chinese & $29(63.0)$ \\
\hline Malay & $5(10.9)$ \\
\hline Indian & $8(17.4)$ \\
\hline Other $^{+}$ & $4(8.7)$ \\
\hline \multicolumn{2}{|c|}{ Mode of emergency department arrival } \\
\hline Self-referral & $1(2.2)$ \\
\hline Ambulance (non-standby) & $3(6.5)$ \\
\hline Ambulance (standby) & $42(91.3)$ \\
\hline \multicolumn{2}{|c|}{ Emergency department presentation after injury } \\
\hline Within $4 \mathrm{hr}$ & $44(95.7)$ \\
\hline After $94 \mathrm{hr}$ & $2(4.3)$ \\
\hline \multicolumn{2}{|l|}{ Mechanism of injury } \\
\hline Blunt & $35(76.1)$ \\
\hline Motor vehicle crash & $24(68.6)$ \\
\hline Fall from height & $10(28.6)$ \\
\hline Direct trauma & $1(2.9)$ \\
\hline Penetrating & $11(23.9)$ \\
\hline Stab & $10(90.9)$ \\
\hline Slash & $1(9.1)$ \\
\hline \multicolumn{2}{|l|}{ Anatomical site of injury } \\
\hline Chest & $6(13.0)$ \\
\hline Abdomen & $2(4.3)$ \\
\hline Thoracoabdominal & $6(13.0)$ \\
\hline Perineal & $1(2.2)$ \\
\hline Multiple ${ }^{\ddagger}$ & $31(67.4)$ \\
\hline
\end{tabular}

*Data presented as median (interquartile range). †Includes Caucasian, Eurasian, Bangladeshi and Filipino patients. ¥Includes injuries to the head, neck and limbs. admission and surgery, had a stab wound to the chest with initial normal CT, but a left-sided pleural effusion persisted despite chest tube drainage. Repeat CTAP revealed a left-sided diaphragmatic tear and herniation. No patient underwent laparoscopic surgery. Among the nine survivors who underwent open surgery, $8(88.9 \%)$ patients had left-sided and $1(11.1 \%)$ had right-sided diaphragmatic herniation. $6(66.7 \%)$ patients were also found to have concomitant solid organ injuries, of whom 3 (50.0\%) had splenic injuries, 2 (33.3\%) had liver injuries and 1 (16.7\%) had multiple solid organ injuries. 5 (55.6\%) patients had concomitant hollow viscus injuries, of whom $2(40.0 \%)$ had perforation of the stomach, $1(20.0 \%)$ had perforation of the small bowel and $2(40.0 \%)$ had laceration of the large bowel. Diaphragmatic herniation was reduced in all nine patients, via simple closure in $8(88.9 \%)$ patients and mesh closure in $1(11.1 \%)$ patient.

Of the patients with acute diaphragmatic herniation who died $(n=5)$, all had suffered blunt trauma (due to motor vehicle crashes [MVCs] in three patients and falls from height for two patients). Their median ISS was 66.0 (IQR 28.5). Of these, $3(60.0 \%)$ patients presented to the ED with traumatic arrest and $2(40.0 \%)$ hypotensive patients died after emergent laparotomies due to other overwhelming injuries. Chest radiography of the two non-arrest patients did not diagnose the diaphragmatic herniation. CT was not done for any of these patients. Autopsies showed left-sided diaphragmatic tears and herniation in all five patients.

Among 22 patients who had diaphragmatic tears without herniation, there were 12 (54.5\%) survivors and 10 (45.5\%) deaths. The median age was 49.0 (IQR 33.0) years. The median ISS of the 12 survivors was 22.0 (IQR 6.5). Chest radiography was performed for all patients but did not diagnose any of the diaphragmatic tears. CT was not performed for 6 (50.0\%) patients, as they were haemodynamically unstable and proceeded to open surgery. CT thorax and CTAP were performed for 2 (16.7\%) and $3(25.0 \%)$ survivors, respectively, while both scans were done for $1(8.3 \%)$ patient. No patient received repeat CT thorax or CTAP. The three CT thorax scans did not reveal any diaphragmatic tears, while $2(50.0 \%)$ CTAP scans revealed right-sided diaphragmatic tears. All 12 patients underwent open surgery $-8(66.7 \%)$ had laparotomy, 3 (25.0\%) had thoracotomy and 1 (8.3\%) underwent both. There were $6(50.0 \%)$ patients each with right- and left-sided diaphragmatic tears. On open surgery, $10(83.3 \%)$ patients had concomitant solid organ injuries: $5(50.0 \%)$ had liver injuries, $1(10.0 \%)$ had lung injuries, $2(20.0 \%)$ had splenic injuries and

Table II. Traumatic diaphragmatic injury subtypes by mechanism of injury.

\begin{tabular}{|c|c|c|c|c|}
\hline \multirow[t]{2}{*}{ Mechanism of injury } & \multicolumn{4}{|c|}{ No. (\%) } \\
\hline & Acute herniation $(n=14)$ & Tear $(n=22)$ & Contusion $(n=10)$ & Total $(n=46)$ \\
\hline \multicolumn{5}{|l|}{ Blunt } \\
\hline Motor vehicle crash & $9(64.3)$ & $9(40.9)$ & $6(60.0)$ & $24(52.2)$ \\
\hline Fall from height & $4(28.6)$ & $2(9.1)$ & $4(40.0)$ & $10(21.7)$ \\
\hline Direct trauma & 0 & $1(4.5)$ & 0 & $1(2.2)$ \\
\hline Stab & $1(7.1)$ & $9(40.9)$ & 0 & $10(21.7)$ \\
\hline Slash & 0 & $1(4.5)$ & 0 & $1(2.2)$ \\
\hline
\end{tabular}


Table III. Comparison of mechanism of injury, mortality rates and Injury Severity Score (ISS) among the three subtypes of traumatic diaphragmatic injuries.

\begin{tabular}{|c|c|c|c|c|}
\hline \multirow[t]{2}{*}{ Variable } & \multicolumn{3}{|c|}{ No. (\%) } & \multirow[t]{2}{*}{ p-value } \\
\hline & Acute herniation $(n=14)$ & Tear $(n=22)$ & Contusion $(n=10)$ & \\
\hline \multicolumn{5}{|c|}{ Mechanism of injury } \\
\hline Blunt & $13(92.9)$ & $12(54.5)$ & $10(100.0)$ & $<0.05$ \\
\hline Penetrating & $1(7.1)$ & $10(45.5)$ & $0(0.0)$ & $<0.05$ \\
\hline Deaths & $5(35.7)$ & $10(45.5)$ & $10(100.0)$ & $<0.05$ \\
\hline \multicolumn{5}{|l|}{ ISS* } \\
\hline Survivor & $34.0(23.0)$ & $22.0(6.5)$ & - & 0.03 \\
\hline Death & $66.0(28.5)$ & $54.0(16.0)$ & $53.5(16.0)$ & 0.77 \\
\hline
\end{tabular}

${ }^{*}$ Data presented as median (interquartile range).

$2(20.0 \%)$ had multiple solid organ injuries. $3(25.0 \%)$ patients also sustained concomitant hollow viscus injuries, with small, large or multiple bowel perforations. All diaphragmatic tears were repaired by simple closure. The median ISS of the patients who died $(\mathrm{n}=10)$ was 54.0 (IQR 16.0). $6(60.0 \%)$ patients had traumatic arrests in the ED, and the remaining 4 (40.0\%) were haemodynamically unstable, receiving chest radiography but no CT. However, the chest radiography did not diagnose the diaphragmatic tears. These four patients underwent laparotomy and $2(50.0 \%)$ also underwent thoracotomy. All patients died within 24 hours postoperatively due to overwhelming multiple injuries. Autopsies were performed for all patients who died, which confirmed the $8(80.0 \%)$ left-sided and $2(20.0 \%)$ rightsided diaphragmatic tears.

All patients with diaphragmatic contusions $(n=10)$ died. Their median age was 30.5 (IQR 46.0) and median ISS was 53.5 (IQR 16.0). All patients had sustained blunt trauma - 6 (60.0\%) from MVCs and $4(40.0 \%)$ due to falls from height. There were $4(40.0 \%)$ patients with traumatic arrests in the ED. The remaining $6(60.0 \%)$ patients were haemodynamically unstable in the ED and required emergency open surgery. They received chest radiography in the $\mathrm{ED}$, but none of the chest radiographs showed the diaphragmatic contusion. Of these six patients, $4(66.7 \%)$ had laparotomy, 1 (16.7\%) had thoracotomy and 1 (16.7\%) had both. On open surgery, 5 (83.3\%) of these patients also had solid organ injuries $-3(60.0 \%)$ had liver injuries, 1 (20.0\%) had lung injuries and 1 (20.0\%) had multiple injuries. 1 (16.7\%) of these six patients also had hollow viscus injury (large bowel laceration). All six patients died within 24 hours postoperatively. On autopsy, all of them were found to have had left-sided diaphragmatic contusions.

Overall, there were 25 (54.3\%) deaths and 21 (45.7\%) survivors till hospital discharge. Table III compares the mechanisms of injury, mortality rates and the median ISS among survivors and deaths for the three TDI subtypes. There were statistically significant differences among patients in each TDI subtype with respect to the mechanism of injury (blunt vs. penetrating), mortality rates and median ISS of survivors.

\section{DISCUSSION}

Our study showed that a different pattern of TDIs was associated with blunt versus penetrating injuries and also highlighted the diagnostic difficulties faced by clinicians when managing these patients and injuries. TDIs were uncommon among patients with trauma at our centre, as captured by our data registry, with only $0.4 \%$ of our trauma patients having TDIs. Our rate of TDIs was lower than the incidence of $0.5 \%-8 \%$ reported in the literature. ${ }^{(3,4)}$ These differences likely reflect the different target populations of trauma patients in the other series, which included patients admitted with blunt trauma ${ }^{(3)}$ and those who required laparotomy or thoracotomy. ${ }^{(4)}$

Similar to other reports, we found that the left hemidiaphragm was more frequently injured. ${ }^{(12,13)}$ This is partly due to the buffering effect of the liver on the right side ${ }^{(14,15)}$ and partly on account of the greater inherent resistance of the right hemidiaphragm relative to the left. ${ }^{(16,17)}$ Blunt trauma was the most common mechanism of injury in our study, as has been reported in other developed countries. ${ }^{(5,6)}$ The most common blunt trauma encountered was from MVCs and falls from height. Stab injuries were the most common penetrating injury leading to TDIs in our study. We did not report any gunshot wounds, which was likely on account of the tight gun control laws in Singapore.

Patients with acute diaphragmatic herniation and contusions in our study were more likely to have sustained these injuries via a blunt mechanism of injury. High-velocity blunt trauma to the abdomen results in a sudden transmission of force through the abdominal viscera that acts as a hydrodynamic fluid wave, leading to significantly increased intra-abdominal pressure and eventual diaphragmatic disruption and herniation. Among patients with diaphragmatic tears, a penetrating mechanism of injury was almost as frequent as a blunt one. Penetrating trauma is more innocuous and produces smaller diaphragmatic defects without herniation. This has a greater possibility of being underrecognised, with patients often showing delayed development of visceral obstruction and strangulation. ${ }^{(18)}$

Chest radiography was the first-line investigation for our patients with acute diaphragmatic herniation who did not present to the ED in the arrest state. However, its efficacy in the diagnosis of TDIs was limited, as it only identified herniation in two out of nine survivors and for none of the patients who died due to the injury. The most definite finding on chest radiography was abdominal viscera in the hemithorax, as seen in two of our patients, or coiling of the nasogastric tube within the left side of the chest. ${ }^{(18)}$ Other findings include the 
absence of fundic gas in its normal position, elevation of the hemidiaphragm and presence of a haemopneumothorax. ${ }^{(19)}$ These later findings are less obvious and can potentially be misinterpreted. Chest radiography was also of limited value in detecting diaphragmatic tears without herniation and contusion, as these injuries were undiagnosed in all the patients who underwent the investigation.

Similarly, CTAP had diagnostic limitations. Many patients in our series did not undergo CT, as they were either haemodynamically unstable or present following traumatic arrest. CTAP could detect TDIs in only four out of seven patients with acute diaphragmatic herniation who underwent $\mathrm{CT}$, giving an estimated sensitivity of $57.1 \%$ in our series. In addition, one of these patients had normal initial CT. Our observation was consistent with the sensitivity of helical CT imaging for diagnosing diaphragmatic herniation, which has been reported to range between $56 \%$ and $87 \%$ in the literature. ${ }^{(20-22)}$ $\mathrm{CT}$, likewise, was limited in diagnosing diaphragmatic tears, detecting TDIs in only two out of six patients who underwent the imaging modality, so that its estimated sensitivity in our series was $33.3 \%$.

In view of the diagnostic limitations of chest radiography and CTAP, our study also highlights the importance of serial assessment in haemodynamically stable patients who do not undergo operative management and show no evidence of TDIs on initial chest radiography or CT. Despite the limitations of CT imaging, we opine that the hospital's policy of selective conservatism in the management of haemodynamically stable and non-gunshot penetrating traumas using $\mathrm{CT}$, examination of trajectory, local wound exploration and clinical observation has been successful in managing TDIs, which can sometimes be clinically challenging to diagnose. This approach has been well described in the literature. ${ }^{(23,24)}$ In our series, we encountered only one patient with delayed diagnosis of acute diaphragmatic herniation from a stab wound to the chest. The injury was suspected following persistent left-sided pleural effusion and confirmed on repeat CT. Our series also showed that if there is an indication for open surgery, the diagnosis of TDI is usually straightforward. All TDIs were treated surgically and mostly by simple closure.

All subtypes of TDIs in our study were associated with significant mortality rates with a range of $35.7 \%-100 \%$. This observation likely demonstrates that TDIs, especially diaphragmatic contusions, were important markers of a severe mechanism of injury resulting in high ISS. These injuries were frequently accompanied by concomitant solid organ and hollow viscus injuries consistent with other reports. ${ }^{(25,26)}$ We did not have patients with chronic diaphragmatic herniation in our series. Nevertheless, a chronic diaphragmatic hernia may present months to years after the initial injury. In the case of blunt trauma, the initial traumatic episode may be long forgotten. ${ }^{(27)}$

This study was not without limitations. As it was performed at a single centre, we may not be able to generalise its findings to other patient populations. In addition, we did not have any patients with TDIs from gunshot wounds. It was also a retrospective study that is subject to recall and reporting biases. However, these biases were minimal, as our trauma database was collected prospectively. The only retrospective information gathering in our study involved searching the national electronic database for any records of re-attendances by our patients after discharge from the traumatic episode due to chronic diaphragmatic herniation. Apart from the limitation of small patient numbers, we might have missed patients with chronic diaphragmatic herniation, especially those that were late entries into the database, who had very short follow-up periods following discharge.

In conclusion, our study revealed varying injury patterns among TDIs, with a mechanism of injury that ranged from blunt to penetrating trauma. TDIs remained a diagnostic challenge and were associated with significant mortality rates, as they were frequently accompanied by concomitant solid organ and hollow viscus injuries. Preoperative chest radiography, CT thorax and CTAP had significant diagnostic limitations. Selective conservatism, as practised at our centre, appeared safe and useful for managing these injuries.

\section{REFERENCES}

1. Agrusa A, Romano G, Chianetta D, et al. Right diaphragmatic injury and lacerated liver during a penetrating abdominal trauma: case report and brief literature review. World J Emerg Surg 2014; 9:33.

2. Vilallonga R, Pastor $\mathrm{V}$, Alvarez $\mathrm{L}$, et al. Right sided diaphragmatic rupture after blunt trauma. An unusual entity. World J Emerg Surg 2011; 6:3.

3. Epstein LI, Lempke RE. Rupture of the right hemidiaphragm due to blunt trauma. J Trauma 1968; 8:19-28.

4. Shah R, Sabaratnam S, Mearns AJ, Choudhury AK. Traumatic rupture of diaphragm. Ann Thorac Surg 1995; 60:1444-9.

5. Matsevych OY. Blunt diaphragmatic rupture: four year's experience. Hernia 2008; $12: 73-8$.

6. Shehata SM, Shabaan BS. Diaphragmatic injuries in children after blunt abdominal trauma. J Pediatr Surg 2006; 41:1727-31.

7. Adegboye VO, Ladipo JK, Adebo OA, Brimmo Al. Diaphragmatic injuries. Afr J Med Med Sci 2002; 31:149-53.

8. Lal S, Kailasia Y, Chouhan S, Gaharwar A, Shrivastava G. Delayed presentation of post traumatic diaphragmatic hernia. J Surg Case Rep 2011; 2011:6.

9. Feliciano DV, Cruse PA, Mattox KL, et al. Delayed diagnosis of injuries to the diaphragm after penetrating wounds. J Trauma 1998; 28:1135-44.

10. Leppäniemi AK, Voutilainen PE, Haapiainen RK. Indications for early mandatory laparotomy in abdominal stab wounds. Br J Surg 1999; 86:76-80.

11. Biffl WL, Kaups KL, Cothren CC, et al. Management of patients with anterior abdominal stab wounds: a Western Trauma Association multicenter trial. J Trauma 2009; 66:1294-301.

12. McGillicuddy D, Rosen P. Diagnostic dilemmas and current controversies in blunt chest trauma. Emerg Med Clin North Am 2007; 25:695-711.

13. Clarke DL, Greatorex B, Oosthuizen GV, Muckart DJ. The spectrum of diaphragmatic injury in a busy metropolitan surgical service. Injury 2009; 40:932-7.

14. Scharff JR, Naunheim KS. Traumatic diaphragmatic injuries. Thorac Surg Clin 2007; 17:81-5.

15. Williams M, Carlin AM, Tyburski JG, et al. Predictors of mortality in patients with traumatic diaphragmatic rupture and associated thoracic and/or abdominal injuries. Am Surg 2004; 70:157-62.

16. Rees O, Mirvis SE, Shanmuganathan K. Multidetector-row CT of right hemidiaphragmatic rupture caused by blunt trauma: a review of 12 cases. Clin Radiol 2005; 60:1280-9.

17. Mirvis SE, Shanmuganathan K. Imaging hemidiaphragmatic injury. Eur Radiol 2007; 17:1411-21.

18. Dwivedi S, Banode P, Gharde P, Bhatt M, Ratanlal Johrapurkar S. Treating traumatic injuries of the diaphragm. J Emerg Trauma Shock 2010; 3:173-6.

19. Yilmaz M, Isik B, Ara C, et al. Gastric perforation during chest tube placement for acute diaphragmatic rupture and review of the literature. Injury Extra 2006; 37:71-5.

20. Nchimi A, Szapiro D, Ghaye B, et al. Helical CT of blunt diaphragmatic rupture. AJR Am J Roentgenol 2005; 184:24-30. 
21. Desser TS, Edwards B, Hunt $\mathrm{S}$, et al. The dangling diaphragm sign: sensitivity and comparison with existing $\mathrm{CT}$ signs of blunt traumatic diaphragmatic rupture. Emerg Radiol 2010; 17:37-44.

22. Allen TL, Cummins BF, Bonk RT, et al. Computed tomography without oral contrast solution for blunt diaphragmatic injuries in abdominal trauma. Am J Emerg Med 2005; 23:253-8.

23. Clarke DL, Thomson SR, Madiba TE, Muckart DJ. Selective conservatism in trauma management: a South African contribution. World J Surg 2005; 29:962-5.
24. Navsaria PH, Berli JU, Edu S, Nicol AJ. Non-operative management of abdominal stab wounds--an analysis of 186 patients. S Afr J Surg 2007; 45:128-30.

25. Shanmuganathan K, Killeen K, Mirvis SE, White CS. Imaging of diaphragmatic injuries. J Thorac Imaging 2000; 15:104-11.

26. Chughtai T, Ali S, Sharkey P, Lins M, Rizoli S. Update on managing diaphragmatic rupture in blunt trauma: a review of 208 consecutive cases. Can J Surg 2009; 52:177-81.

27. Hegarty MM, Bryer JV, Angorn IB, Baker LW. Delayed presentation of traumatic diaphragmatic hernia. Ann Surg 1978; 188:229-33. 